\title{
Nonlinear Identification of Continuous-Time Radio Frequency Power Amplifier Model
}

\author{
Mourad Djamai, Smail Bachir, Claude Duvanaud and Guillaume Mercère
}

\begin{abstract}
In this paper, we present a three-step identification procedure for radio frequency Power Amplifier (PA) in the presence of nonlinear distortion which affect the modulated signal in the Radiocommunication transmission system. The proposed procedure uses a grey box model where PA dynamics are modelled with a MIMO continuous filter and the nonlinear characteristics are described as general polynomial functions, approximated by means of Taylor series. Using the baseband input and output data, model parameters are obtained by an iterative identification algorithm based on Output Error method. Initialization and excitation problems are resolved by an association of a new technique using initial values extraction with a multi-level binary sequence input exciting all PA dynamics. Finally, the proposed estimation method is tested and validated on experimental data.
\end{abstract}

Index Terms-Parameter estimation, power amplifier, continuous time domain, identification algorithm, Output Error technique, initialization problem.

\section{INTRODUCTION}

System identification of High Frequency circuits is of great interest to design complex radiocommunication systems. The exponential growth of the mobile and wireless applications has lead to the development of complex modulation techniques as well as spread spectrum system [1][2]. As a result, non constant envelope signal are used to improve spectral efficiency. The power amplifier, used to transmit the modulated signal, becomes very important in mobile communication systems. This is due to the nonlinear distortions and dynamical effects which caused the increase of the bit error rate and generate unwanted harmonics in the transmitted spectrum signal .

Numerous approaches of modeling PA nonlinearities have been developed in this research area to characterize the input to output complex envelope relationship [3][4]. The model forms used in identification are generally classified into three methods depending on the physical knowledge of the system : black box, grey box and white box [5][6]. A black box model is a system where no physical insight and prior information available. This approach have been widely used in many research studies to predict the output of the Nonlinear Power Amplifier such as neural networks [7][8], Wiener and Volterra series [9][10]. However, this method suffer from the high number of parameters and the time consuming in computation for complex system. On the opposite, white box model is a system where the

S. Bachir, M. Djamai, C. Duvanaud and G. Mercère are with laboratory of Automatic, Electronic and Electrical Engineering, University of Poitiers, 4 avenue de varsovie 16021 Angoulême, France. Email: sbachir@iutang.univpoitiers.fr URL: http://laii.univ-poitiers.fr/ mathematical representation, under some assumptions, is perfectly known. The main advantages of this model are that the resulting parameters have physical significance like gain conversion, damping coefficient and cut-off frequency in electrical systems [11][12]. For many industrial processes, there exists some, but incomplete knowledge concerning the system. This gives a third way of making models of engineering systems: The grey box modelling. This technique describes the model using some ideas about the character of the process that generated the data. For these reasons, the model considered in this paper is a grey box class described in continuous-time domain. This structure is similar to PA discrete-time representation including nonlinear transfer functions and multivariable continuous filter [3][13]. The first block is set to a memoryless complex amplitude (AM/AM) and phase (AM/PM) conversion. Conventionally, a power series model is used to consider these transfer functions. To describe PA dynamics, an $n^{\text {th }}$ MIMO filter is inserted. This element operates on modulating input and represents a lowpass equivalent in envelope signals [14]. With this structure, the electronics engineer can interpret immediately the model in physical terms.

Model parameters are achieved using an iterative identification algorithm based on Output Error method.

During last two decades, there has been a new interest in Output Error techniques [15][16][17]. An overview of approaches is given in [18][19][20]. Output Error (OE) methods are based on iterative minimization of an output error quadratic criterion by a Non Linear Programming (NLP) algorithm. This technique requires much more computation and do not converge to unique optimum. But, OE methods present very attractive features, because the simulation of the output model is based only on the knowledge of the input, so the parameter estimates are unbiased [21][22][23]. Moreover, OE methods can be used to identify non linear systems. For these advantages, the OE methods are more appropriate in microwave systems characterization [11][14]. For PA identification, the parameters initialization and input excitation are very important to ensure global convergence. Then, we propose a new procedure for initialization search based on estimation of the nonlinear (AM/AM) and (AM/PM) functions decoupled from filter identification. A resulting value gives a good approximation of model parameters. Associated with a multi-level input excitation, this technique allows a fast convergence to the optimal values. Such an identification procedure for continuous-time domain in PA modeling does not seem to have been used previously. 
The validation of this PA model is obtained for some experimental digital modulation techniques. Measured and estimated output signal are compared. Results show a good agreement and the possibility to PA characterization using continuous-time representation.

\section{PA MODEL DESCRIPTION}

The nonlinear amplifier model used in this paper is an extension of the discrete time-model at continuous representation [3][13][24]. The major disadvantage of the discrete representation is that the used parameters have no physical significance, contrary to continuous one where parameters keep their real aspect [16][25]. This is very important when advanced PA applications are considered such as linearization or real time control.

The nonlinear block presented here operates on baseband quadrature I/Q time-domain waveforms. The complex lowpass equivalent (LPE) representation of the communication signal is used to avoid the high sampling rate required at the carrier frequency.

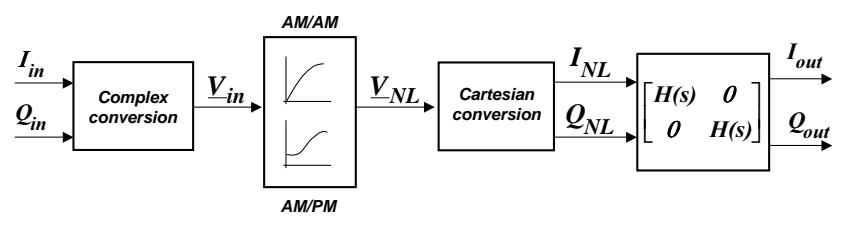

Fig. 1. Radio frequency power amplifier model

As shown in fig. 1, the two-box MIMO model includes a memoryless nonlinearity prior to an $n^{\text {th }}$ order Laplace filter. In this model, the first box is the AM/AM and AM/PM conversions described PA nonlinearities. The second box is the frequency response which operates on the two baseband inputs $I / Q$.

\section{A. Nonlinear Static functions}

To take into account simultaneous gain and phase characteristics, amplifiers are traditionally modeled with a complex polynomial series [9]. Then, the complex envelope of the non linear output signal is approximated with the following baseband input/output relationship:

$$
\underline{V}_{N L}=\underline{V}_{i n} \cdot G\left(\left|\underline{V}_{i n}\right|^{2}\right)
$$

$\underline{V}_{i n}$ and $\underline{V}_{N L}$ are respectively the complex input and output voltage translated in baseband and expressed according the direct and quadrature $I / Q$ signals as:

$$
\left\{\begin{array}{lll}
\underline{V}_{i n} & =I_{i n}+j Q_{i n} \\
\underline{V}_{N L} & =I_{N L}+j Q_{N L}
\end{array}\right.
$$

$G\left(\left|\underline{V}_{i n}\right|^{2}\right)$ is the complex gain of the amplifier, dependent of the magnitude of the input $\underline{V}_{i n}$. The complex gain is expressed with a polynomial function composed by even term which produce harmonic distortions inside the PA bandpass:

$$
G\left(\left|\underline{V}_{i n}\right|^{2}\right)=\sum_{k=0}^{P} \underline{c}_{2 k+1} \cdot\left|\underline{V}_{i n}\right|^{2 k}
$$

where $\underline{c}_{2 k+1}$ are the complex power series coefficients such as:

$$
\underline{c}_{2 k+1}=\alpha_{2 k+1}+j \beta_{2 k+1}
$$

The previous equations give the relationship between input and output baseband signals :

$$
\left\{\begin{array}{l}
\underline{I}_{N L}=\sum_{k=0}^{P}\left(\alpha_{2 k+1} I_{i n}-\beta_{2 k+1} Q_{i n}\right) \cdot\left|\underline{V}_{i n}\right|^{2 k} \\
\underline{Q}_{N L}=\sum_{k=0}^{P}\left(\alpha_{2 k+1} Q_{i n}+\beta_{2 k+1} I_{i n}\right) \cdot\left|\underline{V}_{i n}\right|^{2 k}
\end{array}\right.
$$

The output quadrature signals depend on the both input quadrature terms and on the instantaneous input power.

\section{B. Continuous filter}

The dynamical effect caused by the PA system behavior may be expressed with a differential equation. As shown in figure (1), the input to output relationships of this $n^{\text {th }}$ order filter can be written as:

$$
\left\{\begin{array}{l}
\frac{d^{n}}{d t^{n}} I_{\text {out }}+\sum_{k=0}^{n-1} a_{k} \frac{d^{k}}{d t^{k}} I_{\text {out }}=\sum_{k=0}^{m} b_{k} \frac{d^{k}}{d t^{k}} I_{N L} \\
\frac{d^{n}}{d t^{n}} Q_{\text {out }}+\sum_{k=0}^{n-1} a_{k} \frac{d^{k}}{d t^{k}} Q_{\text {out }}=\sum_{k=0}^{m} b_{k} \frac{d^{k}}{d t^{k}} Q_{N L}
\end{array}\right.
$$

where $I_{\text {out }}(t)$ and $Q_{\text {out }}(t)$ are the filter outputs.

The coefficients $\left\{a_{k}\right\}$ and $\left\{b_{k}\right\}$ are real scalars that define the model. Note that the filter structure is the same on the two axes $I$ and $Q$, which gives a decoupled MIMO plant. Thus, the input-output relation can be expressed in Laplace-domain with the transfer-function $H(s)$, as so:

$$
H(s)=\frac{\sum_{k=0}^{m} b_{k} \cdot s^{k}}{s^{n}+\sum_{k=0}^{n-1} a_{k} s^{k}}
$$

where $s$ denotes the differential operator $s=\frac{d}{d t}$.

\section{PARAMETER IDENTIFICATION OF THE PA MODEL}

The problem of system identification is a major field in control and signal processing [22]. For their simplicity, the Equation Error (EE) techniques like least squares are regarded as the most suitable methods for estimating the coefficients in a regression model. However, there are severe drawbacks, not acceptable in PA characterization, especially for the identification of physical parameters, such as the residual error caused by the output noise and the modeling errors [12].

Output Error (OE) methods have become a wide-spread technique for non linear system identification [21][23]. Usually, for these methods, parameter estimates are found iteratively using optimization algorithms. The simulation of the output model is based only on the knowledge of the input, so the parameter estimation is unbiased [22]. 


\section{A. Identification algorithm}

Parameter identification is based on the definition of a model. For power amplifier, we consider the previous mathematical model (Eqs. 1-7) and we define the parameter vector:

$$
\underline{\theta}=\left[a_{0} \cdots a_{n-1} b_{0} \cdots b_{m} \underline{c}_{1} \cdots \underline{c}_{2 P+1}\right]^{T}
$$

where [.] ${ }^{T}$ denotes transposition operation.

Assume that we have measured $K$ values of input vector $\left(I_{\text {in }}(t), Q_{\text {in }}(t)\right)$ and output vector $\left(I_{\text {out }}^{*}(t), Q_{\text {out }}^{*}(t)\right)$ with $t=k$. $T_{e}$ and $1 / T_{e}$ is the sampling rate. The identification problem is then to estimate the values of the parameters $\underline{\theta}$. Thus, we define the output prediction errors:

$$
\left\{\begin{array}{l}
\varepsilon_{I_{k}}=I_{\text {out }_{k}}^{*}-\hat{I}_{\text {out }_{k}}\left(\hat{\hat{\theta}}, I_{\text {in }}, Q_{\text {in }}\right) \\
\varepsilon_{Q_{k}}=Q_{\text {out }_{k}}^{*}-\hat{Q}_{\text {out }_{k}}\left(\underline{\hat{\theta}}, I_{\text {in }}, Q_{i n}\right)
\end{array}\right.
$$

where predicted outputs $\hat{I}_{\text {out }_{k}}$ and $\hat{Q}_{\text {out }_{k}}$ are obtained by numerical simulations of the PA model and $\underline{\hat{\theta}}$ is an estimation of true parameter vector $\underline{\theta}$.

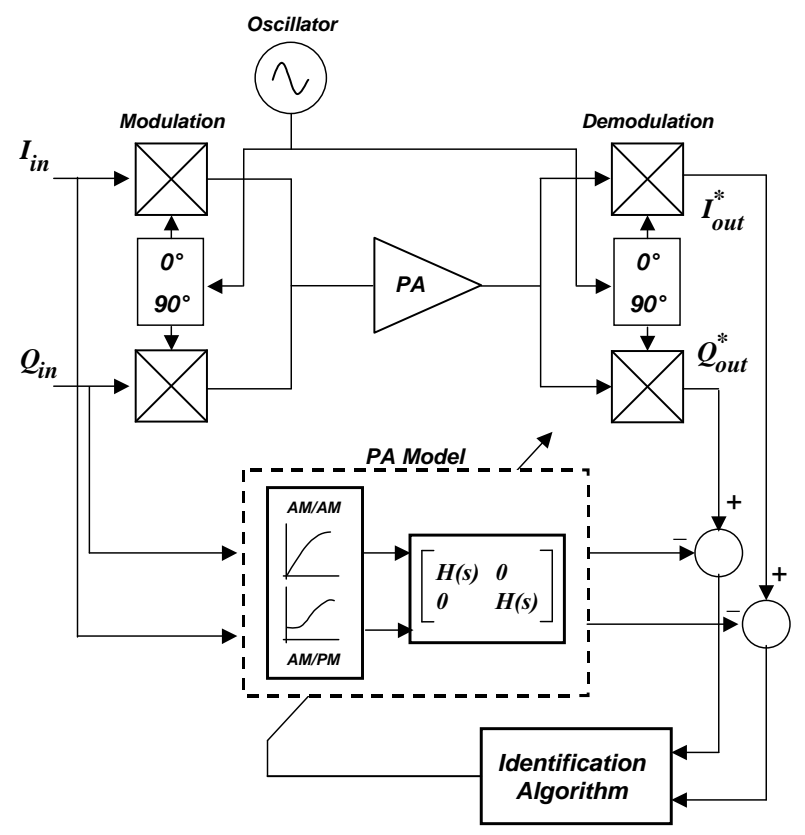

Fig. 2. PA identification scheme

As a general rule, parameter estimation with $\mathrm{OE}$ technique is based on minimization of a quadratic multivariable criterion defined as :

$$
J=\sum_{k=1}^{K}\left(\varepsilon_{I k}^{2}+\varepsilon_{Q_{k}^{2}}^{2}\right)=\underline{\varepsilon}_{I}^{T} \underline{\varepsilon}_{I}+\underline{\varepsilon}_{Q}^{T} \underline{\varepsilon}_{Q}
$$

We obtain the optimal values of $\underline{\theta}$ by Non Linear Programming techniques. Practically, we use Marquardt's algorithm [28] for off-line estimation:

$$
\underline{\hat{\theta}}_{i+1}=\underline{\hat{\theta}}_{i}-\left\{\left[J_{\theta \theta}^{\prime \prime}+\lambda \cdot I\right]^{-1} \cdot J_{\theta}^{\prime \prime}\right\}_{\hat{\hat{\theta}}=\underline{\theta}_{i}}
$$

$J_{\theta}^{\prime}$ and $J_{\theta \theta}^{\prime \prime}$ are respectively gradient and hessian such as:
$J_{\theta}^{\prime}=-2 \sum_{k=1}^{K}\left(\underline{\varepsilon}_{I_{k}}^{T} \cdot \underline{\sigma}_{I_{k, \underline{\theta}}}+\underline{\varepsilon}_{Q_{k}}^{T} \cdot \underline{\sigma}_{Q_{k, \underline{\theta}}}\right)$

$J_{\theta \theta}^{\prime \prime} \approx 2 \sum_{k=1}^{K}\left(\underline{\sigma}_{I_{k, \underline{\theta}}} \cdot \underline{\sigma}_{I_{k, \underline{\theta}}}^{T}+\underline{\sigma}_{Q_{k, \underline{\theta}}} \cdot \underline{\sigma}_{Q_{k, \underline{\theta}}}^{T}\right)$

$\lambda$ is the monitoring parameter,

$\underline{\sigma}_{I_{k, \underline{\theta}}}=\frac{\partial \hat{\underline{I}}_{\text {out }}}{\partial \underline{\underline{\theta}}}$ an output sensitivity on $I$ axis,

and $\underline{\sigma}_{Q_{k, \underline{\theta}}}=\frac{\partial \hat{\hat{Q}}_{\text {out }}}{\partial \underline{\underline{\theta}}}$ an output sensitivity on $Q$ axis.

\section{B. Sensitivity functions}

The sensitivity functions $\underline{\sigma}$ are important elements in the identification procedure. The positive realness of these functions ensures the stability and the convergence of an unbiased identification algorithm. In comparison, sensitivity functions are equivalent to the regressors in the linear case [12]. Thus, it is necessary to attach a great importance to the calculation of these functions.

For nonlinear grey-box identification, consider a general continuous-time state-space model structure :

$$
\begin{aligned}
& \dot{\dot{x}}(t)=\underline{g}(\underline{x}(t), \underline{\theta}, u(t)) \\
& y(t)=f(\underline{x}(t), \underline{\theta}, u(t))
\end{aligned}
$$

where $g$ and $f$ are nonlinear functions. $x(t)$ is the state vector $(\operatorname{dim}(\underline{x})=N), u(t)$ and $y(t)$ are input and output signals, and $t$ denotes time. Finally $\underline{\theta}$ is the vector of unknown parameters $(\operatorname{dim}(\underline{\theta})=I)$.

We consider a SISO non-linear system only to simplify the equations; there is no restrictive assumption on the dimensions of $\underline{u}$ and $\underline{y}$.

In the proposed formulation, it is necessary to distinguish two kinds of sensitivity functions:

- $\underline{\sigma}_{y, \underline{\theta}}=\frac{\partial y}{\partial \underline{\theta}}$ : vector of output sensitivity functions $(I \times 1)$ used in the NLP algorithm - $\sigma_{\underline{x}, \underline{\theta}}=\frac{\partial \underline{x}}{\partial \underline{\theta}}: \begin{aligned} & \text { matrix of state sensitivity functions }(N \times I) \\ & \text { such as: }\end{aligned}$

$$
\sigma_{\underline{x}, \underline{\theta}}=\left[\begin{array}{lllll}
\underline{\sigma}_{\underline{x}, \theta_{1}} & \cdots & \underline{\sigma}_{\underline{x}, \theta_{i}} & \cdots & \underline{\sigma}_{\underline{x}, \theta_{I}}
\end{array}\right]
$$

The sensitivity functions $\sigma_{x, \theta_{i}}$ are obtained, for each parameter $\theta_{i}$, by partial differentiation of equation (12). Thus

$$
\frac{\partial \underline{\dot{x}}}{\partial \theta_{i}}=\underline{\dot{\sigma}}_{\underline{x}, \theta_{i}}=\frac{\partial g(\underline{x}, \underline{\theta}, u)}{\partial \underline{x}} \frac{\partial \underline{x}}{\partial \theta_{i}}+\frac{\partial g(\underline{x}, \underline{\theta}, u)}{\partial \theta_{i}}
$$

So, $\sigma_{\underline{x}, \theta_{i}}$ is the solution of the nonlinear differential system:

$$
\underline{\dot{\sigma}}_{\underline{x}, \theta_{i}}=\frac{\partial g(\underline{x}, \underline{\theta}, u)}{\partial \underline{x}} \underline{\sigma}_{\underline{x}, \theta_{i}}+\frac{\partial g(\underline{x}, \underline{\theta}, u)}{\partial \theta_{i}}
$$

Finally, we obtain the output sensitivity functions used in Non Linear Programming algorithm by partial differentiation of equation (13), we get:

$$
\sigma_{y, \theta_{i}}=\frac{\partial y}{\partial \theta_{i}}=\left(\frac{\partial f(\underline{x}, \underline{\theta}, u)}{\partial \underline{x}}\right)^{T} \underline{\sigma}_{\underline{x}, \theta_{i}}+\frac{\partial f(\underline{x}, \underline{\theta}, u)}{\partial \theta_{i}}
$$


In the particular case of linear system described by the following state space model :

$$
\left\{\begin{array}{l}
\dot{x}=A(\underline{\theta}) \underline{x}+B(\underline{\theta}) u \\
y=C^{T}(\underline{\theta}) \underline{x}+D(\underline{\theta}) u
\end{array}\right.
$$

we obtain :

$$
\left\{\begin{array}{c}
\underline{\dot{\sigma}}_{\underline{x}, \theta_{i}}=A(\underline{\theta}) \underline{\sigma}_{\underline{x}, \theta_{i}}+\left[\frac{\partial A(\underline{\theta})}{\partial \theta_{i}}\right] \underline{x}+\left[\frac{\partial B(\underline{\theta})}{\partial \theta_{i}}\right] u \\
\sigma_{y, \theta_{i}}=C^{T}(\underline{\theta}) \underline{\sigma}_{\underline{x}, \theta_{i}}+\left[\frac{\partial C(\underline{\theta})}{\partial \theta_{i}}\right]^{T} \underline{x}+\left[\frac{\partial D(\underline{\theta})}{\partial \theta_{i}}\right] u
\end{array}\right.
$$

All discrete-time models are deduced from the continuous one by second order serie expansion of the transition matrix.

\section{Initialization problems}

An inherent problem of iterative search routines is that only convergence to a local minimum can be guaranteed. In order to converge to the global minimum, a good initial parameter estimate is important. Usually, for engineering process, users have a good knowledge on physical parameters, necessary to initialize the iterative algorithm (Eq. 11). In our case, PA users have not sufficient information on parameter vector $\underline{\theta}$, especially on AM/AM and AM/PM parameters. It is then essential to define a global strategy which makes it possible to obtain approximative values of parameters. So we propose an optimal search method based on Equation Error techniques to achieve initial values of non linear and filter parameters.

\section{1) Non linear parameters initialization:}

The first step consists in searching approximation of the complex parameters $\underline{c}_{2 k+1}$ using the envelope magnitude and phase distorsions (Eqs. 1-3). Thus, the AM/AM and AM/PM characteristics are used to optimize a polynomial function by Least Mean Square (LMS) algorithm [22]. A solution for the coefficients is obtained by minimizing the mean-squared error between the measured $\left(I_{\text {out }}^{*}, Q_{\text {out }}^{*}\right)$ and the modeled output $\left(I_{\text {out }}, Q_{\text {out }}\right)$ under low frequency signal such as:

$$
\underline{\hat{\theta}}_{N L}=\left(\phi^{H} \phi\right)^{-1} \phi^{H} \underline{V}_{o u t}^{*}
$$

where :

$(.)^{H}$ denotes transpose-conjugate transformation

$\underline{\hat{\theta}}_{N L}=\left[\begin{array}{llll}\underline{c}_{1} & \underline{c}_{3} & \cdots & \underline{c}_{2 P+1}\end{array}\right]^{T}$ is the vector of polynomial parameters,

$\underline{V}_{\text {out }}^{*}$ is the measured output,

$\phi=\left[\begin{array}{llll}\underline{\varphi}_{1} & \underline{\varphi}_{2} & \cdots & \underline{\varphi}_{K}\end{array}\right]$ is the regression matrix,

$\underline{\varphi}_{k}=\left[\begin{array}{llll}V_{i n_{k}} & V_{i n_{k}}\left|V_{i n_{k}}\right|^{2} & \cdots & V_{i n_{k}}\left|V_{i n_{k}}\right|^{2 P}\end{array}\right]^{T}$ is the regression vector,

and $\underline{V}_{i n_{k}}$ is a $k^{\text {th }}$ sampled input.

Noted that for these estimations, the regression vector $\underline{\varphi}_{k}$ is not correlated with the measured output $\underline{V}_{\text {out }}^{*}$.

In practice, the PA characteristics is performed by a sinusoidal excitation applied on baseband inputs $I_{i n}$ and $Q_{i n}$ at fixed low frequency and high input level. In these conditions, the PA filtering effects are assumed negligible according to non linear dynamics. The input-output curves are obtained by measuring the output gain and phase as a function of input level.

\section{2) Filter parameters initialization:}

The second step is the determination of initial values for the filter coefficients. They are obtained for an input signal with low input level and large frequency bandwidth. The signal distorsion is then negligible, which makes it possible to take into account only the linear filter effects. Thus, we define the filter parameter vector:

$$
\underline{\theta}_{f}=\left[\begin{array}{llllllll}
a_{0} & a_{1} & \cdots & a_{n-1} & b_{0} & b_{1} & \cdots & b_{m}
\end{array}\right]^{T}
$$

Parameter estimation is performed by iterative Instrumental Variable based on Reinitilized Partial Moments RPM method (see also [12][14][17]). Used for continuous filter identification, this technique is included in the integral methods class. The main idea of this class is to avoid the input-output timederivatives calculation by performing integrations. In this class, the particularities of the RPM method ${ }^{1}$ is the use of a time-shifting window for the integration and to perform an output noise filtering.

The main advantage of this estimation method to others is its relatively insensitivity to the initial conditions and rough system a priori knowledge.

\section{PA SETUP}

The measurement setup is shown in Fig. 3. The power amplifier is a commercial ZHL-42 from MINI CIRCUITS manufacturer. Input and output data are obtained from YOKOGAWA DIGITAL OSCILLOSCOPE with a sampling period equal to $10 \mathrm{~ns}$.

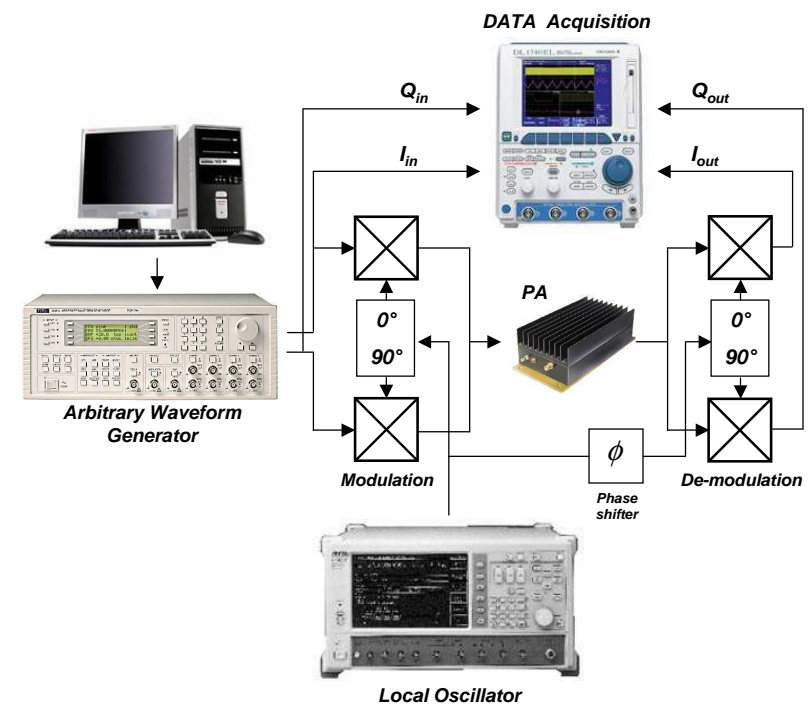

Fig. 3. PA setup

\footnotetext{
${ }^{1}$ Contsid Matlab TOOLBOX including the RPM estimation method can be downloaded from http://www.cran.uhp-nancy.fr/contsid/. The ivrpm function allows to obtain model estimation by iterative Instrumental Variable.
} 
Filter identification algorithm needs large frequency bandwidth excitation signal to provide appropriate estimation. Indeed, modulated signals are required to excite both steadystate (low frequency) and process dynamics (medium to high frequency). This excitation is performed with a Pseudo Random Binary Sequence (P.R.B.S) baseband pulse as the input modulation to the transmitter. All data processing are carried using MATLAB MATHWORKS then are downloading to a BASEBAND WAVEFORM GENERATOR. The quadrature modulator AD8349 and demodulator AD8347 are inserted at the input and output of the PA. They are standard commercial units from Analog Devices.

Modulation signals $I$ and $Q$ are delivered by a TT $i 40 \mathrm{MHz}$ Arbitrary Waveform Generator connected to PC control. The local oscillator frequency is $900 \mathrm{MHz}$ obtained from Digital Modulation Signal Generator (ANRITSU MG 3660A).

The identification procedure is performed in three steps : Initialization of nonlinear parameters, initialization of filter parameter and global identification of the PA's model.

\section{A. Experimental results}

Nonlinear parameters $\underline{c}_{k}$ are extracted from the input/output transfer function. The AM/AM and AM/PM measured characteristics are obtained by sweeping the power level of an input signal at a frequency located at the center of the PA bandwidth. In our case, we used the $3^{\text {th }}$ order complex polynomial:

$$
\underline{V}_{N L}=\left(\underline{c}_{1}+\underline{c}_{3} \cdot\left|\underline{V}_{i n}\right|^{2}+\underline{c}_{5} \cdot\left|\underline{V}_{i n}\right|^{4}\right) \cdot \underline{V}_{i n}
$$

Thus, we define the estimated parameter complex vector:

$$
\underline{\theta}_{N L}=\left[\begin{array}{lll}
\underline{c}_{1} & \underline{c}_{3} & \underline{c}_{5}
\end{array}\right]^{T}
$$

After running a $L M S$ algorithm (Eq. 19), we obtained :

$$
\left\{\begin{array}{l}
\hat{\underline{c}}_{1}=1.222-0.115 j \\
\hat{\hat{c}}_{3}=-0.0918+0.0299 j \\
\underline{\hat{c}}_{5}=0.01710^{-2}-0.06210^{-2} j
\end{array}\right.
$$
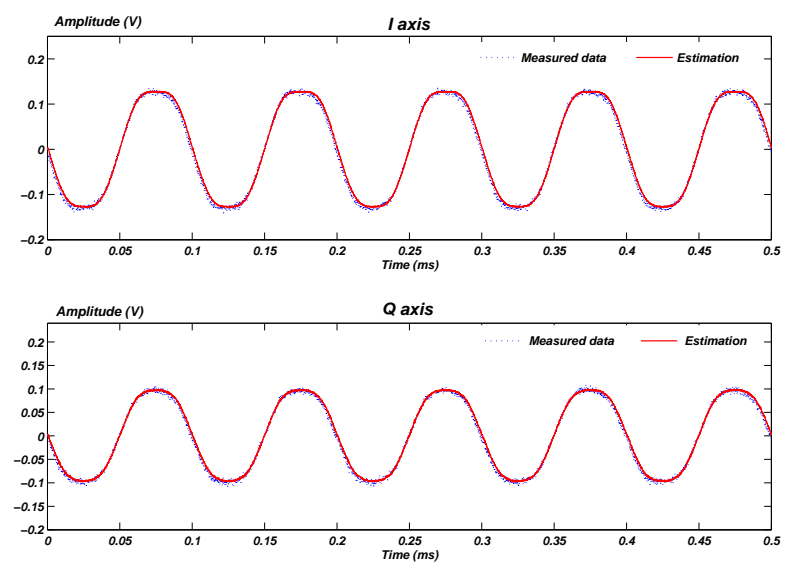

Fig. 4. Comparison of time-domain measurement and estimation
Figure (4) allows a comparison between measured $I$ and $Q$ outputs waveforms and their estimations. As can be seen, even if the amplifier is driven near saturation, the LMS algorithm converge to the optimum values with a maximum output estimation error less than $0.008 \mathrm{~V}$.

$\mathrm{AM} / \mathrm{AM}$ and $\mathrm{AM} / \mathrm{PM}$ characteristics are given in figure (5). Thus, we can clearly see that the non linear behavioral of the amplifier is successfully described by a traditional third polynomial series.
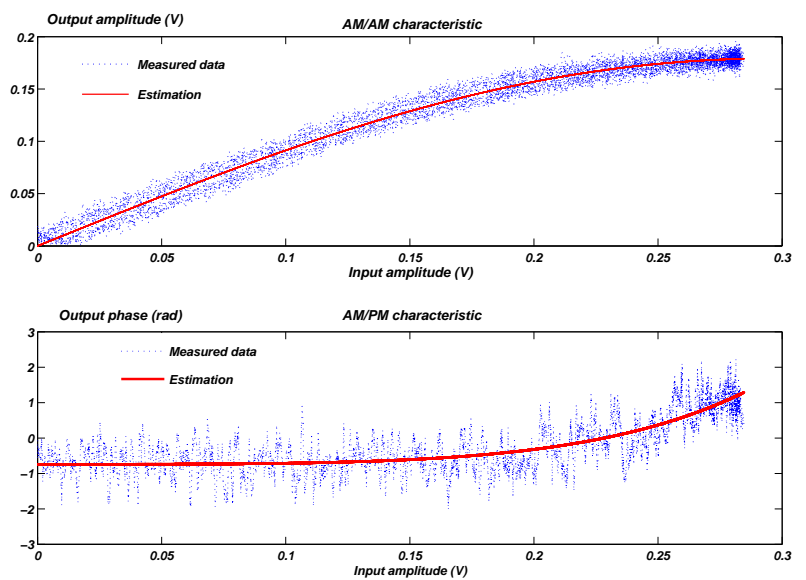

Fig. 5. Comparison between the measured and estimated AM/AM and AM/PM functions
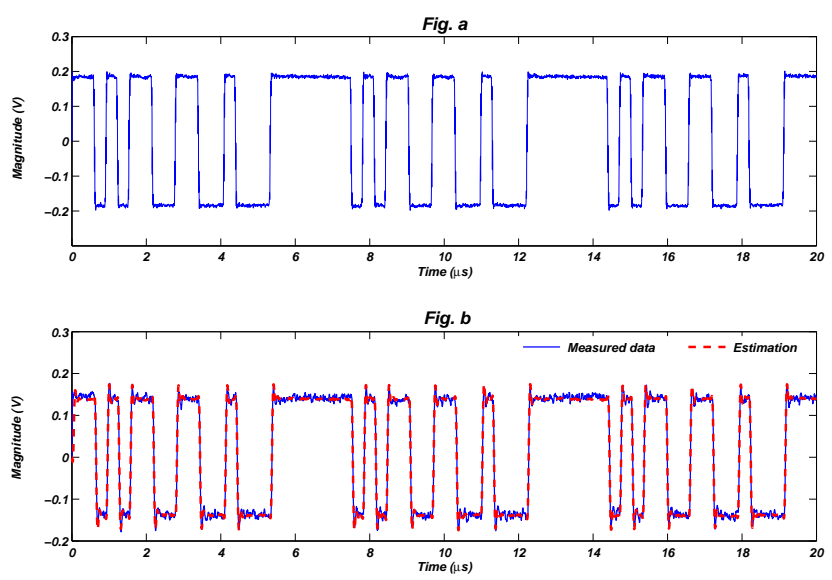

Fig. 6. (a) Input signal. (b) Comparison of time-domain measurement and estimation

The initial values of the linear filter parameters are obtained by applying a Pseudo Random Binary Sequence $(P R B S)$ signal with small amplitude level. The filter form is achieved using the RPM method for different plants. A quadratic error comparison allows to obtain an appropriate order. Then, the $3^{\text {rd }}$ order filter are defined in the Laplace domain as:

$$
H(s)=\frac{b_{1} s+b_{0}}{s^{3}+a_{2} s^{2}+a_{1} s+a_{0}}
$$


Thus, we define the estimated parameter vector:

$$
\underline{\theta}_{f}=\left[\begin{array}{lllll}
a_{0} & a_{1} & a_{2} & b_{0} & b_{1}
\end{array}\right]^{T}
$$

The RPM algorithm gives the parameters values:

$$
\left\{\begin{array}{l}
\hat{a}_{0}=2.01 \cdot 10^{23} \\
\hat{a}_{1}=6.11 \cdot 10^{23} \\
\hat{a}_{2}=9.60 \cdot 10^{7} \\
\hat{b}_{0}=1.51 \cdot 10^{23} \\
\hat{b}_{1}=-1.79 \cdot 10^{15}
\end{array}\right.
$$

For small power, figure (6) shows that the PA dynamic can be modeled as a $3^{\text {rd }}$ order resonant system.

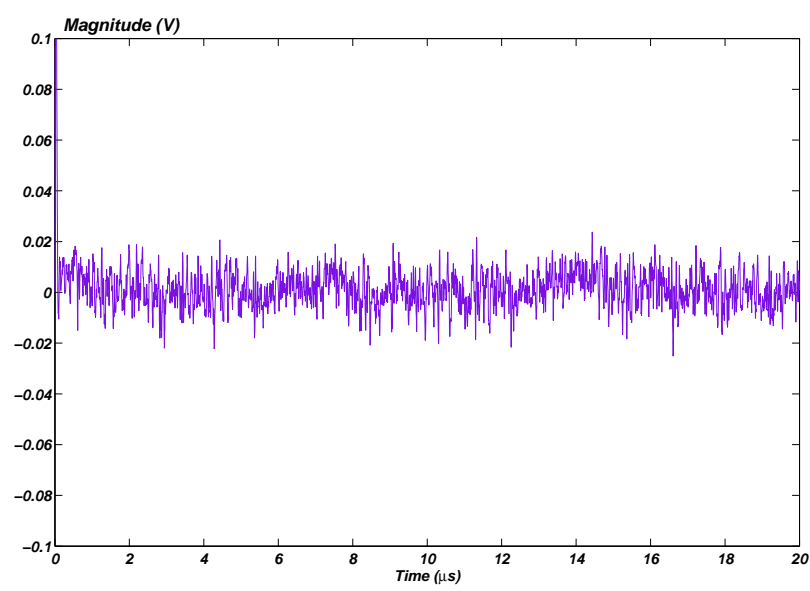

Fig. 7. Identification residuals

As observed in figure (7), the identification residuals (estimation error) are negligible and dont exceed $0,04 \mathrm{~V}$.

The dynamic behavioral of the PA system can be described by a MIMO filter. The filter characteristic is represented in figure (8) by the gain and phase curves. The resonnant frequency of the filter is around $9.8 \mathrm{MHz}$.

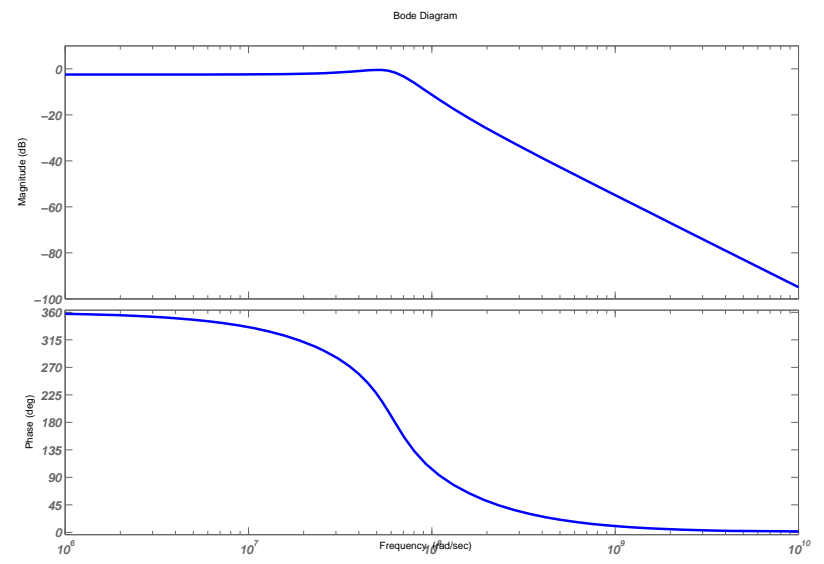

Fig. 8. Frequency responses of the PA gain and the phase

\section{B. PA global identification :}

The model parameters obtained in the previous section will be used to initialize the nonlinear identification algorithm. The unknown system in this case is the global PA model composed by both:

- non-linear complex polynomial functions.

- $3^{\text {rd }}$ order filter system.

The measurements are performed by an input signal obtained from the adding of some P.R.B.Sequences at different levels. The aim is to drive the amplifier in its overall level range (linear and non linear area). Figure (9.a) shows the input signal applied to perform global PA identification. After 8 iterations, we obtain the following parameters:

$$
\begin{gathered}
\hat{\underline{\theta}}_{N L}=\left\{\begin{array}{l}
\hat{\underline{c}}_{1}=1.181+8.452 \cdot 10^{-3} j \\
\hat{\underline{c}}_{3}=-0.042-0.023 j \\
\underline{\hat{c}}_{5}=-0.201 \cdot 10^{-2}+0.316 \cdot 10^{-2} j
\end{array}\right. \\
\underline{\hat{\theta}}_{f}=\left\{\begin{array}{l}
\hat{a}_{0}=2.01 \cdot 10^{23} \\
\hat{a}_{1}=6.11 \cdot 10^{15} \\
\hat{a}_{2}=9.65 \cdot 10^{7} \\
\hat{b}_{0}=1.51 \cdot 10^{23} \\
\hat{b}_{1}=-1.79 \cdot 10^{15}
\end{array}\right.
\end{gathered}
$$
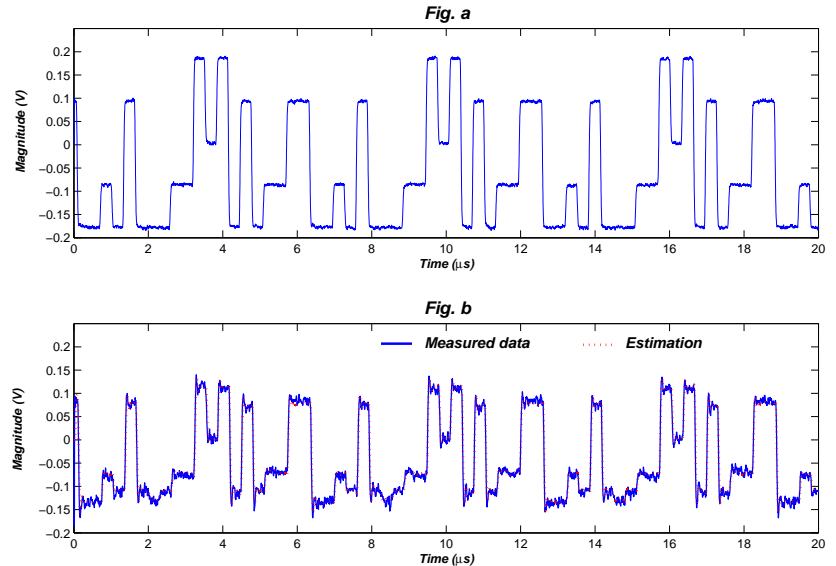

Fig. 9. (a) Input signal. (b) Comparison of time-domain measurement and estimation

Model simulation with the achieved parameters exhibit good approximation of measured data (fig. 9.b).

\section{Model validation}

In this section, we validate the PA model by comparing predicted and measured outputs for different modulation schemes. As a test signal, we use a QPSK digitally modulated signal shaped with a raised cosine filter with a Rolloff factor of $\alpha=0.25$.

Figures (10.a) and (10.b) compare the simulated model output (dotted line) with the measured output for an excitation signal different of the one previously used for identification (solid line). It can be seen that the simulated output follows the measured one. 

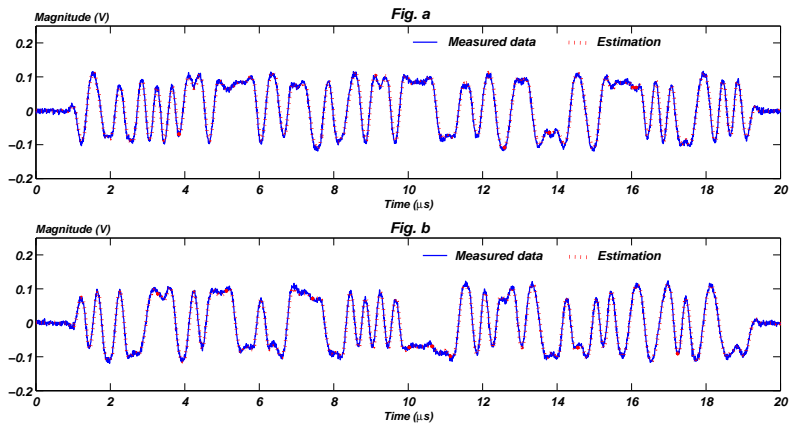

Fig. 10. Comparison of I/Q time-domain measurement and estimation for QPSK modulation

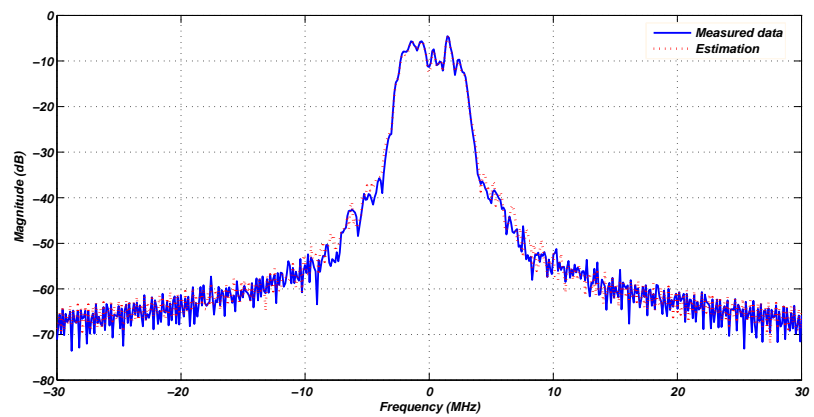

Fig. 11. Measured and estimated output spectrum

To validate the proposed model, figure (11) compares the measured and simulated output power spectral densities at specific frequencies.

\section{CONCLUSION}

A model based on continuous-time representation is described which offers a simple way to modeling PA dynamics. This model is able of accounting the magnitude and phase amplifier nonlinearities such as the saturation effects.

Test results illustrate the efficiency of this technique for use in off-line identification. The continuous approach was found to be accurate in predicting the dynamical response of the power amplifier. Estimation results show that the described amplifier acts like a resonant system coupled with a polynomial series.

The proposed technique is based on continuous time domain model. The model achieved can be used to develop a continuous baseband method for the compensation of nonlinearity of the RF front-end in a wireless transmitter.

\section{REFERENCES}

[1] S. C. Cripps, Advanced Techniques in RF power Amplifier Design, Artech House, Boston 2002.

[2] P. B. Kennington, High-linearity RF Amplifier Design, Artech House, Boston 2000.

[3] C. J. Clark, G. Chrisikos, M. S. Muha, A. A. Moulthrop and C. P. Silva, Time-domain envelope measurement technique with application to wideband power amplifier modeling, in IEEE Trans. Microwave Theory Tech. VOL. 46, NO. 12, pp. 2531-2540, Dec. 1998.
[4] M. Isakson, D. Wisell and D. Ronnow, A Comparative Analysis of behavioral Models for RF Power Amplifiers, in IEEE Trans. Microwave Theory Tech., VOL. 54, No. 1, pp. 348-359, Junuary 2006.

[5] L . Ljung, Some aspects on nonlinear system identification, IFAC Symposium on System Identification SYSID'06, Australia, 2006.

[6] H. Tulleken, Grey-box modeling and identification using physical knowledge and bayesian techniques, Automatica, VOL. 29, N0. 02, pp. 285-308, 1993.

[7] T. Liu, S. Boumaiza, and F. M. Ghannouchi, Dynamic Behavioral modeling of $3 G$ Power Amplifiers Using real-Valued Time-Delay Neural Networks, in IEEE Trans. Microwave Theory Tech., VOL. 52, No. 3, pp. 1025-1033, March 2004.

[8] J. Xu, M. C. E. Yagoub, R. Ding and Q. J. Zhang, Neural-Based Dynamic Modeling of Nonlinear Microwave Circuits, in IEEE Trans. Microwave Theory Tech., VOL. 50, No. 12, pp. 2769-2780, December 2002.

[9] F. Launay, Y. Wang, S. Toutain, D. Barataud, J. M. Nebus and R. Quere, Nonlinear amplifier modeling taking into account HF memory frequency, in MTT-S Int. Micro. Symposium Digest, VOL. 02, pp. 865-868, 2002.

[10] A. Zhu, M. Wren, T. J. Brazil, An Efficient Volterra-based Behavioral Model for Wideband RF Power Amplifiers, MTT-S Int. Microwave Symposium Digest, VOL. 02, pp. 865- 868, 2003.

[11] M. Djamai, S. Bachir and C. Duvanaud, Behavioral modeling and digital predistortion of RF power amplifiers, Integrated Nonlinear Microwave and Millimetre-wave Circuits (INMMiC), Portugal, January 2006.

[12] J. C. Trigeassou, T. Poinot and S. Bachir, Méthodes de commande des machines électriques, Chapter 7 : Estimation paramétrique pour la connaissance et le diagnostic des machines électriques, pp. 215251, Traité EGEN - Série G. Electrique, Hermès Publications 2003.

[13] P. Stoica and T. Söderstrom, Instrumental-variable methods for identification of hammerstein systems, International Journal of Control, VOL. 35, NO. 3, pp. 459-476, 1982.

[14] M. Djamai, E. Tohme, S. Bachir and R. Ouvrard, Continuous-Time Model Identification Using Reinitialized Partial Moments - Application To Pwer Amplifier Modeling, IFAC Symposium on System Identification SYSID'06, Australia, 2006.

[15] E. Walter and L. Pronzato, Identification of parametric models from experimental data, Communication and Control Engineering Series, Editions Springer, 1997.

[16] H. Unbehauen and G.P. Rao, A review of identification in continuoustime systems, Annual Reviews in Control 22, pp. 145-171, 1998.

[17] M. Mensler, H. Garnier and E. Huselstein, Experimental comparison of continuous-time model identification methods on thermal process, In: 12th IFAC Symposium on System Identification. Santa Barbara, California 2000.

[18] I. D. Landau, Adaptive Control : The model reference aproach, Marcel Dekker. Control and Systems Theory. VOL. 08, 1979.

[19] P. Young, Parameter estimation for continuous-time models - a survey, Automatica, VOL. 17, No. 01, pp. 23-39, 1981.

[20] H. Unbehauen and G.P. Rao, Identification of continuous systems, Systems and control series. North-Holland, 1987.

[21] J. Richalet, A. Rault and R. Pouliquen, Identification des processus par la mèthode du modèle, Gordon \& Breach, Thérie des systèmes, $\mathrm{N}^{o} .04,1971$.

[22] L. Ljung, System identification: Theory for the user, Prentice Hall, USA, 1987.

[23] J. C. Trigeassou, Recherche de modèles expérimentaux assistée par ordinateur, Technique et Documentation Lavoisier, Paris, 1988.

[24] J. Vörös, Parameter identification of Wienner systems with discontinuous nonlinearities, Systems and Control Letters, VOL. 44, pp. 363-372, 2001.

[25] V. Peterka, Trends and Progress in System Identification, Chapter Bayesian approach to system identification, pp. 239-304, Editions P. Eykhoff, Pergamon, Oxford, 1981.

[26] H. F. Chen and L. Guo, Identification and Stochastic Adaptive Control, Birkhauser, Boston, 1991.

[27] N. B. Carvalho and J. C. Pedro, A Comprehensive explanation of distortion sideband asymmetries, in IEEE Trans. Micro. Theo. Tech., VOL. 50, pp. 2090-2101, 2002.

[28] D. W. Marquardt, An Algorithm for least-squares estimation of nonlinear parameters, Soc. Indust. Appl. Math, VOL. 11, NO. 2, pp. 431-441, 1963. 